\title{
Electroencephalography Patterns of Subacute Sclerosing Panencephalitis
}

\author{
Saad Ali ${ }^{1}$, Harwindar Kumar ${ }^{1}$, Shakir Ullah ${ }^{2}$, Mian Ayaz U. Haq ${ }^{1}$, Nusrat G. Gul ${ }^{3}$, Jasvindar Kumar ${ }^{4}$ \\ 1. Neurology, Lady Reading Hospital Medical Teaching Institute Peshawar, Peshawar, PAK 2. Pharmacology, Khyber \\ Medical University, Peshawar, PAK 3. Neurophysiology, Lady Reading Hospital Medical Teaching Institute Peshawar, \\ Peshawar, PAK 4. Internal Medicine, Khyber Teaching Hospital, Peshawar, PAK
}

Corresponding author: Harwindar Kumar, kumar281@hotmail.com

\begin{abstract}
Introduction and background: This article explores the electroencephalography (EEG) pattern in patients of suspected subacute sclerosing panencephalitis (SSPE) visiting Lady Reading Hospital, Peshawar. Pakistan has a huge deficit of research culture, and limited studies are done on this topic. This study concluded that a typical pattern is the most common EEG pattern, although atypical and normal EEG patterns were also observed. It is worth inclusion into the existing literature and may be used for future literature review of similar studies done elsewhere in Pakistan to give a wider perspective comprised of a larger sample size integrating all studies.
\end{abstract}

Objective: To determine the frequency of different patterns of EEG (typical, atypical, and normal) in SSPE patients.

Methodology: Seventy-seven patients of both male and female genders between ages one to 20 years, who were diagnosed with SSPE, were included in the study. Dyken's criteria were used to diagnose the patients. A prior history of previous measles infection with signs and symptoms suggestive of SSPE and positive antimeasles IgG antibodies in the cerebrospinal fluid (CSF) was found in all the patients included in the study. Besides this, typical EEG patterns and raised CSF globulin levels were also used for confirmation of the diagnosis. All the patients fulfilling the above criteria and presenting to the neurology department of Lady Reading Hospital, Peshawar, from February 1, 2019, to November 30, 2019, were included in the study. All the patients underwent the EEG monitoring in the same EEG laboratory and were reported by the same consultant with careful exclusion of any artifacts during the study.

Result: There were 59 (76.62\%) males and 18 (23.37\%) females. The mean age was $15 \pm 8.6$ years, and the mean duration of symptoms was $4.79 \pm 1.68$ months. EEG was normal in $14(18.18 \%)$ patients, while 63 (81.81\%) patients had an abnormal EEG pattern, with a majority of $53(84.12 \%)$ patients showing periodic delta wave complexes. Only $10(15.87 \%)$ patients showed atypical patterns.

Review began 05/26/2021 Review ended 06/16/2021 Published 06/17/2021

() Copyright 2021 Ali et al. This is an open access article distributed under the terms of the Creative Commons Attribution License CC-BY 4.0., which permits unrestricted use, distribution, and reproduction in any medium, provided the original author and source are credited.
Conclusion: Almost all the patients of SSPE showed periodic high-amplitude delta waves complexes, which usually occurs in patients with a disease duration of more than four months. However, further studies with a large sample size are needed for the confirmation of this observation.

Categories: Neurology, Pediatrics, Infectious Disease

Keywords: electroencephalography, subacute sclerosing panencephalitis, dyken's criteria, complication of measles, neurodegenerative disorder

\section{Introduction}

Subacute sclerosing panencephalitis (SSPE) is a chronic neurodegenerative disease that is a post-infection sequela of measles [1]. The estimated frequency of SSPE post measles in the United States is 8.5 per million cases of measles [2]. In the developed world, measles and its complications are rare, but recent evidence suggests that it is more common than previously thought. The incidence of measles was noted to be one in 1367 and one in 609 in children aged <five years and < 12 months, respectively. Different studies from different parts of the world suggested the incidence varies from one in 700 to one in 25,000 in the patients of measles [3-6].

SSPE usually presents with the symptoms of motor involvement such as jerking movements, muscle spasms, ataxia, tremor, seizure, etc. [7]. However, several atypical presentations are also noted [8-11]. Post-measles neurological complications include acute encephalitis, measles inclusion body encephalitis, acute disseminated encephalomyelitis (ADEM), and SSPE. SSPE has a different time and course than ADEM [12]. SSPE is quite a fatal disease in developing countries mainly due to lack of early diagnosis and no proper treatment. Some type of medical treatment in the form of isoprinosine, ribavirin, and interferon-alpha can be offered to the patients who are diagnosed at an early stage of the disease. Various diagnostic tools such as measles antibody detection in cerebrospinal fluid (CSF) and serum, neuroimaging, and EEG are routinely 


\section{Cureus}

used for the investigation of SSPE. Among these, EEG is the simplest, non-invasive, and cheapest investigation with easy availability.

The EEG findings of SSPE are usually shown to be the periodic complexes that are high-amplitude delta waves and are repeated every four to seven seconds [12]. This EEG pattern occurs in almost all the patients at some stage of the disease [12]. Some atypical EEG patterns, which includes asymmetric periodic complexes, spike and slow waves pattern, and paroxysmal rhythmic delta activity between periodic complexes, are also seen in this disease. The long-term video-split EEG has improved the early diagnosis of this disease.

This study is designed to evaluate the cases of SSPE for the different patterns of EEG. In developing countries like Pakistan where measles and its consequences are still not only prevalent but also quite fatal, the knowledge of different EEG patterns will help in the early diagnosis of the patients.

\section{Materials And Methods}

Patients from both genders aged between one and 20 years who were diagnosed with SSPE were enrolled in the study. These patients presented between February 1, 2019, to November 30, 2019, to the neurology department of Lady Reading Hospital, Peshawar. All the patients were diagnosed with SSPE using Dyken's criteria.

Patients with a family history of seizure, neurological problems, and meningeal signs were excluded from the study. Duration of symptoms was noted since the motor involvement. The diagnosis of SSPE was based on the history of prior measles infection, clinical features, serum and CSF measles antibody levels, CSF immunoglobulin levels, and neuroimaging. Most of the patients were having mood swings and personality changes followed by focal neurological features such as jerking movement, muscle spasms, seizure, loss of vision, etc. Neuroimaging was normal in some of the patients, but the majority had occipital white matter changes. Serum and CSF anti-measles antibody were sent to the lab, and those with anti-measles IgG antibodies in cerebrospinal fluid were taken as positive. All the patients underwent the EEG monitoring in the same EEG lab and were reported by the same consultant with careful exclusion of any artifacts during the study.

Periodic delta wave complexes were composed of slow waves in the delta range with a high amplitude (200$500 \mathrm{mV}$ ), symmetrical and bisynchronous. These waves were repeatedly seen every $4-10$ seconds. The other atypical patterns observed were asymmetric periodic complexes, spikes, and spike-slow wave complexes.

Data were analyzed through Statistical Package for the Social Sciences (SPSS) version 20 (IBM Corp., Armonk, NY, USA). The percentages, frequency, mean, standard deviation (SD), and p values were calculated and presented in tables in this article.

\section{Results}

Out of 77 patients, 59 (76.62\%) were males and 18 (23.37\%) were females. The mean age was $15 \pm 8.6$ years, and the mean duration of symptoms was $4.79 \pm 1.68$ months (Table 1 ).

\begin{tabular}{|c|c|c|}
\hline & & Values \\
\hline \multirow{2}{*}{ Gender } & Male & $59(16.62 \%)$ \\
\hline & Female & $18(23.37 \%)$ \\
\hline Age (years) & & $15 \pm 8.6$ \\
\hline Duration of symptoms (months) & & $4.79 \pm 1.68$ \\
\hline
\end{tabular}

TABLE 1: Baseline characteristics $(n=77)$

EEG was normal in 14 (18.18\%) patients, while 63 (81.81\%) patients had an abnormal EEG finding (Table 2 ). Among these, 53 (84.12\%) patients showed typical periodic slow wave complexes (Figure 1), while 10 patients (15.18\%) had atypical patterns (Table 3). This included asymmetric periodic complexes, spike, and slow waves pattern (Figure 2) as well as paroxysmal rhythmic delta activity between periodic complexes as $60 \%$, $30 \%$, and $10 \%$, respectively (Table 4 ). 


\section{Cureus}

EEG findings

Abnormal EEG

Normal EEG
Number of patients

$63(81.81 \%)$

$14(18.18 \%)$

TABLE 2: EEG findings $(n=77)$

EEG, Electroencephalography.

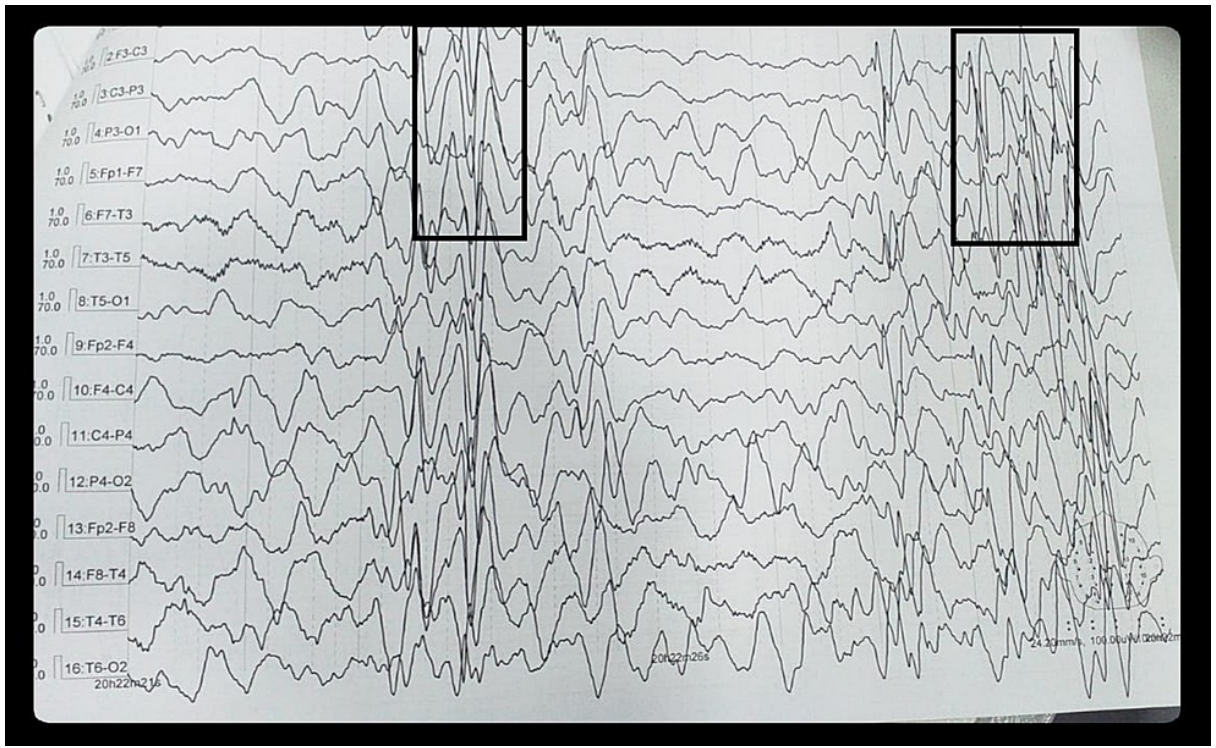

FIGURE 1: Symmetrical periodic slow wave complexes (typical pattern)

EEG findings

Typical symmetrical periodic slow wave complexes

Atypical patterns
Number of patients

$53(84.12 \%)$

$10(15.87 \%)$

TABLE 3: Abnormal EEG findings $(n=63)$

EEG, Electroencephalography. 


\section{Cureus}

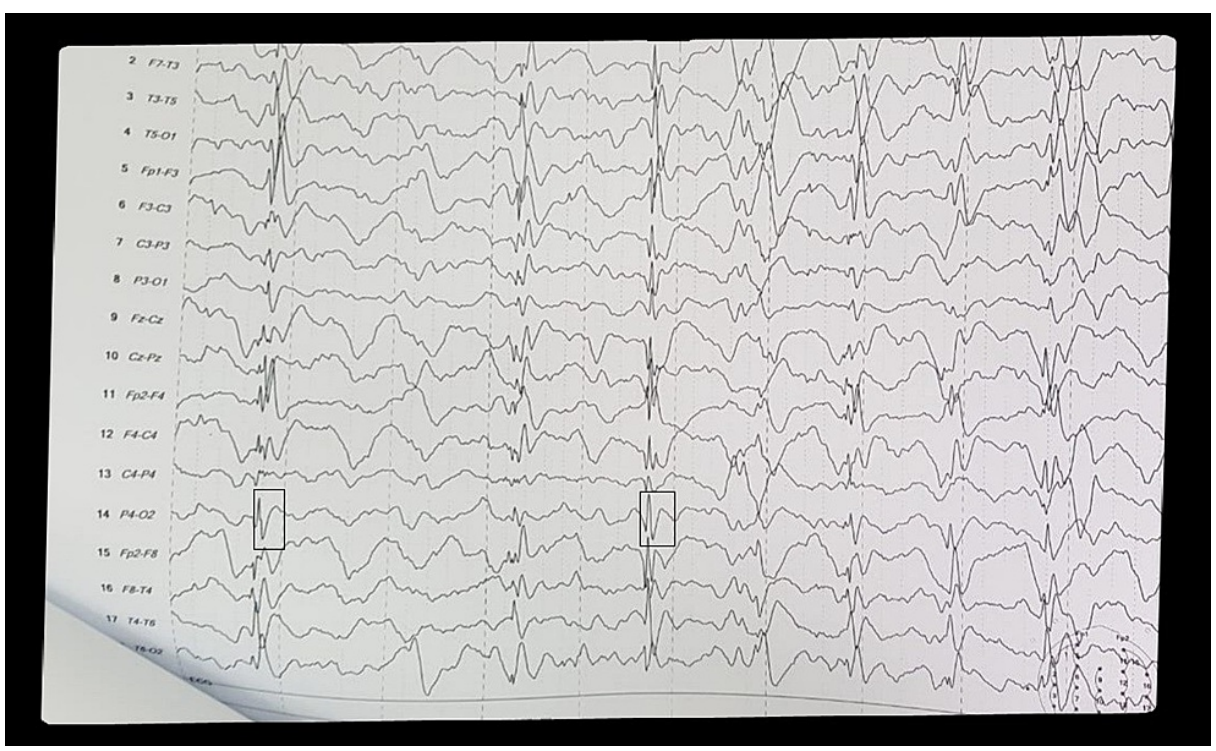

FIGURE 2: Spike and slow waves (atypical pattern)

Types of atypical patterns

Asymmetric periodic complexes

Spike and slow waves pattern

Paroxysmal rhythmic delta activity between periodic complexes
Number of patients

$6(60 \%)$

$3(30 \%)$

$1(10 \%)$

TABLE 4: Types of atypical patterns included $(n=10)$

The duration of symptoms affected the patterns of EEG. There was a positive correlation between the duration of symptoms and the occurrence of periodic delta waves (typical pattern) $(p$-value $=0.125)$ (Table 5).

\begin{tabular}{|c|c|c|c|c|c|}
\hline & & \multicolumn{3}{|l|}{ EEG } & \multirow[b]{2}{*}{ P values } \\
\hline & & Normal EEG & Periodic delta wave complexes & Atypical pattern & \\
\hline \multirow{2}{*}{ Gender } & Male & $11(18.64 \%)$ & $32(54.23 \%)$ & $16(27.11 \%)$ & \multirow{2}{*}{0.641} \\
\hline & Female & $3(16.66 \%)$ & $7(38.88 \%)$ & $8(44.44 \%)$ & \\
\hline \multirow{2}{*}{ Age groups } & $<12$-years old & $11(21.56 \%)$ & $28(45.09 \%)$ & $17(33.33 \%)$ & \multirow{2}{*}{0.154} \\
\hline & >12-years old & $3(14.28 \%)$ & $10(47.61 \%)$ & 8 (38.09\%) & \\
\hline \multirow{2}{*}{ Duration groups } & $<4$ months of symptoms & $4(5.19 \%)$ & $13(16.88 \%)$ & $4(5.19 \%)$ & \multirow{2}{*}{0.125} \\
\hline & $>4$ months of symptoms & $10(12.98 \%)$ & 40 (51.94\%) & $6(7.79 \%)$ & \\
\hline
\end{tabular}

TABLE 5: Association of demographic features and duration of symptoms against EEG pattern (n = 77)

EEG, Electroencephalography.

In our results, SSPE was found more in males (76.62\%) as compared to females (23.37\%) with a ratio of 3.27 to 1 . 


\section{Discussion}

Measles is the most contagious virus yet known, and it is still an important cause of childhood mortality and blindness in developing countries as well as sporadic outbreaks in the industrialized nation [13]. Vaccination against measles is effective, cost-effective, and safe [14]. It is almost six decades since vaccination for the prevention of measles has been licensed [15]. However, most of the population in developing and underdeveloped countries remained unvaccinated because of which measles and its complications are still seen in clinical practice. It requires a high level of population immunity to interrupt the transmission of measles; therefore, it is difficult to eliminate in the high-population density areas [16].

Dyken's criteria are often used as the diagnostic tool for SSPE; this includes clinical history, elevated CSF measles antibody titers, typical EEG pattern, increased CSF immunoglobulin G (IgG), and brain biopsy.

Measles causes four major central nervous system (CNS) syndromes: acute encephalitis, post-viral encephalomyelitis, measles inclusion body encephalitis, and subacute sclerosing panencephalitis (SSPE) [17]. Symptoms of SSPE typically present about eight to 11 years of post-measles infection [18]. The chances of SSPE are higher if measles is acquired earlier in life $[2,6]$. Individuals infected with the human immunodeficiency virus (HIV) or those who are born to mothers with HIV infection might be at higher risk of developing SSPE after measles infection $[19,20]$.

Currently, there is no treatment for SSPE, and eradication by an effective vaccination program is considered to be most beneficial and cost-effective than the medications considered for the management of SSPE [21].

SSPE is usually a life-threatening disease with no cure, despite the development of antiviral and immunomodulatory drugs. A multicentered non-randomized study of 98 patients with SSPE treated with isoprinosine reported increased survival for over two years in the intervention group [22]. SSPE is caused by particular mutants of the measles virus, which are often referred to as the SSPE virus [23].

In our study, we found that most patients (63 out of 77) with SSPE had an abnormal EEG, and the most common abnormality was periodic high-amplitude delta waves complexes that occurred in 53 patients (79.4\%) and the atypical patterns that occurred in 10 patients (15.87\%). The study done by Praveen-kumar et al. showed a typical pattern in 37 patients and atypical patterns in 21 patients [24]. Another study by Wulff et al. found periodic complexes in all patients of SSPE [25]. Our results correlated with all these studies.

There was no association between age and pattern of EEG in our study. The mean age in our study was $15 \pm$ 8.6 years. However, maturation to posterior alpha rhythm occurs around 12 years of age; therefore, there may be some bias over here.

The duration of the disease showed a significant relation to periodic delta wave complexes as with the onset of motor symptoms. The majority (51.94\%) of patients with motor symptoms after more than four months of disease onset had positive EEG for periodic high-amplitude delta waves. As the disease is quite fatal in developing countries, the cases with a longer duration from the time of onset were not seen. Other studies in which long-term duration was noted showed that after some years in disease progression the cortical damage occurs, and the EEG changes, then almost disappear [25].

The shorter duration of disease in our study was an important limitation that limited us from showing the EEG pattern in advanced disease. Another limitation was evaluation of only the type I periodic highamplitude complexes; the other type II and type III complexes were not evaluated, and it might have merged in the atypical pattern. Furthermore, due to one institution focused, shorter duration of symptoms, and small sample size, the result cannot be generalized to all SSPE patients. For these reasons, another study with a large number of patients is needed.

\section{Conclusions}

Almost all the patients of SSPE showed periodic high-amplitude delta waves that usually occurred in patients with a disease duration of more than four months. This study also confirms the SSPE ratio of male to female (3:1), which has been shown by other studies. In addition, the typical pattern of EEG is more common in SSPE; however, we should also keep in mind that atypical and normal patterns of EEG can also occur in SSPE. In the developing world with limited resources, EEG can be used as a good diagnostic tool for the suspected cases of SSPE.

\section{Additional Information}

\section{Disclosures}

Human subjects: Consent was obtained or waived by all participants in this study. Animal subjects: All authors have confirmed that this study did not involve animal subjects or tissue. Conflicts of interest: In compliance with the ICMJE uniform disclosure form, all authors declare the following: Payment/services info: All authors have declared that no financial support was received from any organization for the 
submitted work. Financial relationships: All authors have declared that they have no financial relationships at present or within the previous three years with any organizations that might have an interest in the submitted work. Other relationships: All authors have declared that there are no other relationships or activities that could appear to have influenced the submitted work.

\section{References}

1. Bellini WJ, Rota JS, Lowe LE, et al.: Subacute sclerosing panencephalitis: more cases of this fatal disease are prevented by measles immunization than was previously recognized. J Infect Dis. 2005, 192:1686-93. $10.1086 / 497169$

2. Wendorf KA, Winter K, Zipprich J, et al.: Subacute sclerosing panencephalitis: the devastating measles complication that might be more common than previously estimated. Clin Infect Dis. 2017, 65:226-32. 10.1093/cid/cix302

3. Farrington CP: Subacute sclerosing panencephalitis in England and Wales: transient effects and risk estimates. Stat Med. 1991, 10:1733-44. 10.1002/sim.4780101111

4. Miller C, Andrews N, Rush M, Munro H, Jin L, Miller E: The epidemiology of subacute sclerosing panencephalitis in England and Wales 1990-2002. Arch Dis Child. 2004, 89:1145-8. 10.1136/adc.2003.038489

5. Miller C, Farrington CP, Harbert K: The epidemiology of subacute sclerosing panencephalitis in England and Wales 1970-1989. Int J Epidemiol. 1992, 21:998-1006. 10.1093/ije/21.5.998

6. Schönberger K, Ludwig M-S, Wildner M, Weissbrich B: Epidemiology of subacute sclerosing panencephalitis (SSPE) in Germany from 2003 to 2009: a risk estimation. PLoS One. 2013, 8:e68909. 10.1371/journal.pone.0068909

7. Rota PA, Rota JS, Goodson JL: Subacute sclerosing panencephalitis. Clin Infect Dis. 2017, 65:233-234. 10.1093/cid/cix307

8. Martins R, Peres J, Casimiro C, Valverde A: Subacute sclerosing panencephalitis presenting as rapidly progressive dementia in a young adult. Neurologia (Engl Ed). 2018, 33:206-7. 10.1016/j.nrl.2017.01.009

9. Vural E, Engin E, Demir N, Agan K, Midi I: Unusual progression of an adult-onset subacute sclerosing panencephalitis (SSPE) in Turkey. Epilepsy Behav Rep. 2019, 12:100332. 10.1016/j.ebr.2019.100332

10. Parmar A, Ranjan R, Sagar R: Subacute sclerosing panencephalitis presenting with isolated positive psychotic and catatonic symptoms. Indian J Psychol Med. 2017, 39:534-6. 10.4103/0253-7176.211756

11. Bhat BA, Dar SA, Hussain A: Sub-acute sclerosing panencephalitis presenting as anti-depressant induced childhood mania. J Psychiatry. 2018, 21: 1000452. 10.4172/2378-5756.1000452

12. Markand ON, Panszi JG: The electroencephalogram in subacute sclerosing panencephalitis . Arch Neurol. 1975, 32:719-26. 10.1001/archneur.1975.00490530041002

13. Fiebelkorn AP, Redd SB, Gallagher K, Rota PA, Rota J, Bellini W, Seward J: Measles in the United States during the postelimination era. J Infect Dis. 2010, 202:1520-8. 10.1086/656914

14. Bester JC: Measles and measles vaccination: a review. JAMA Pediatr. 2016, 170:1209-15. 10.1001/jamapediatrics.2016.1787

15. Perry RT, Halsey NA: The clinical significance of measles: a review . J Infect Dis. 2004, 189:S4-16. $10.1086 / 377712$

16. Moss WJ, Griffin DE: Global measles elimination. Nat Rev Microbiol. 2006, 4:900-8. 10.1038/nrmicro1550

17. Fisher DL, Defres S, Solomon T: Measles-induced encephalitis. QJM. 2015, 108:177-82. 10.1093/qjmed/hcu113

18. Upadhyayula PS, Yang J, Yue JK, Ciacci JD: Subacute sclerosing panencephalitis of the brainstem as a clinical entity. Med Sci (Basel). 2017, 5:26. 10.3390/medsci5040026

19. Kija E, Ndondo A, Spittal G, Hardie DR, Eley B, Wilmshurst JM: Subacute sclerosing panencephalitis in South African children following the measles outbreak between 2009 and 2011. S Afr Med J. 2015, 105:7138. 10.7196/SAMJnew.7788

20. Manesh A, Moorthy M, Bandopadhyay R, Rupali P: HIV-associated sub-acute sclerosing panencephalitis - an emerging threat?. Int J STD AIDS. 2017, 28:937-9. 10.1177/0956462416687675

21. Garg RK: Subacute sclerosing panencephalitis. J Neurol. 2008, 255:1861-71. 10.1007/s00415-008-0032-6

22. Jones C, Huttenlocher P, Dyken P, Jabbour JT, Maxwell K: Inosiplex therapy in subacute sclerosing panencephalitis: a multicentre, non-randomised study in 98 patients. Lancet. 1982, 1:1034-7. 10.1016/s0140-6736(82)92097-9

23. Hotta H, Jiang DP, Nagano-Fujii M: [SSPE virus and pathogenesis]. Nihon Rinsho. 2007, 65:1475-80.

24. Praveen-kumar S, Sinha S, Taly AB, Jayasree S, Ravi V, Vijayan J, Ravishankar S: Electroencephalographic and imaging profile in a subacute sclerosing panencephalitis (SSPE) cohort: a correlative study. Clin Neurophysiol. 2007, 118:1947-54. 10.1016/j.clinph.2007.06.008

25. Wulff CH: Subacute sclerosing panencephalitis: serial electroencephalographic studies . J Neurol Neurosurg Psychiatry. 1982, 45:418-21. 10.1136/jnnp.45.5.418 